\title{
The relationship between non-high density lipoprotein cholesterol and insuline resistance in obese patients
}

Authors Melih Macit ${ }^{1}$, Meral Mert² ${ }^{2}$ Ramazan Ateş${ }^{1}$, Özlem Polat ${ }^{1}$,Sadık Sami Hatipoglư ${ }^{3}$

Hospital $\quad{ }^{1}$ Bakırköy Dr Sadi Konuk Educational and Research Hospital, Family Medicine

${ }^{2}$ Bakırköy Dr Sadi Konuk Educational and Research Hospital, Endocrinology and Metabolism,

${ }^{3}$ Bakırköy Dr Sadi Konuk Educational and Research Hospital, Pediatrics

\section{Objectives:}

Obesity is defined as the

presence of excessive fat in the body.

Insulin resistance (IR), type-2 diabetes, dyslipidemia, hypertension and the risk of atherosclerosis increase depending on obesity.

\section{Reducing the} cardiovascular disease (CVD) risk is one of the objectives of the treatment in modality to the obese patient. Therefore, the correction of IR and dyslipidemic conditions of patients is important for the prevention of CVD.

The aim of our study is to determine the relationship between IR and non-high-density lipoprotein cholesterol (non-HDL-C) in patients that come to our hospital due to obesity.

\section{Results:}

In these research totally 248 patients, 35 male $(14,1 \%)$ and 213 female $(85,9 \%)$ with ages ranging from 18 to 89 , were included. When male and female patients are compared, significant differences were observed only for the parameters of HDL-C and TG $(p<0,05)$. While it is found that HDL-C is higher in female group, TG is higher in male group. Intra-group comparisons revealth at there is a highly significant correlation between HOMA-IR and HDL-C $(p<0.01)$. It is also observed that HOMA-IR and TG are highly correlated with each other among women $(p<0.01)$. Among women it is also found that non-HDL-C is highly correlated both with $\mathrm{FG}$ and $\mathrm{HbA} 1 \mathrm{c}$ $(p<0.01)$.
Between January 2012 to June 2015, 248 patients over the age of eighteen, non pregnant, followed with a diagnosis of obesity but did not receive the treatment of metformin and statin are investigated retrospectively in Bakırkoy Dr. Sadi Konuk Training and Research Hospital Endocrinology Clinic.

Fasting glucose (FG), HbA1c, insulin, HOMA-IR (homeostasis model assessment of IR), low-density lipoprotein cholesterol (LDL-C), triglycerides (TG), high-density lipoprotein cholesterol (HDL-C), total cholesterol (TC) and non-HDL-C parameters of these patients were evaluated. Non-HDL $C$ was calculated using the formula total cholestherol- HDL cholesterole.

SPSS-20 was used as statistical analysis. MannWhitney $U$ test was used for the group differences.

When HOMA-IR as insulin resistance marker was found related with HDL-C and TG in women, the absence of this relation in men may be due to number of patients. However, the absence of correlation between HOMA-IR and non-HDL-C in male and female patients may be because of a more strong relationship between HOMAIR and TG in early period.

In addition to this, during this period the fact that non-HDL-C is highly correlated with both $\mathrm{HbA} 1 \mathrm{c}$ and FG level may suggest that the importance of non-HDL-C in prediabetes and diabetes stages may increase. 